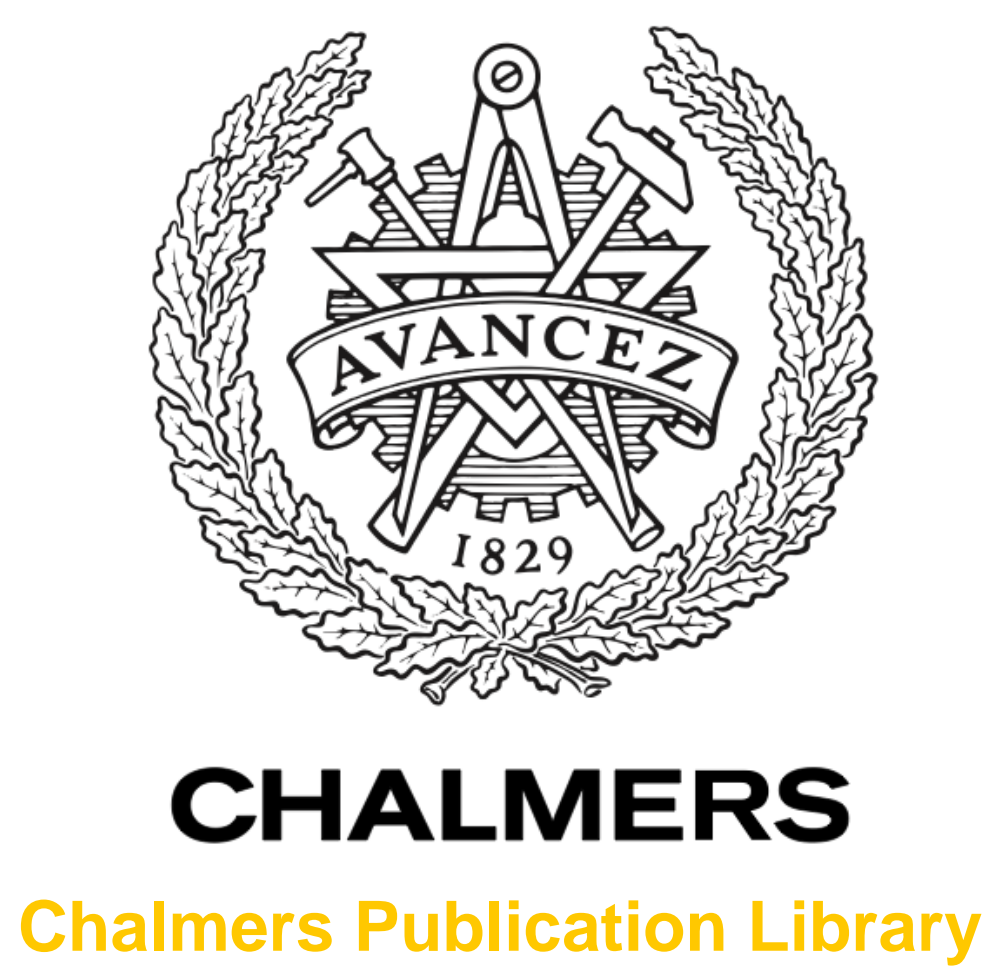

\title{
Fabricationtion and Characteristics of Mesh Band-Pass Filters
}

This document has been downloaded from Chalmers Publication Library (CPL). It is the author's version of a work that was accepted for publication in:

Instruments and Experimental Techniques (ISSN: 0020-4412)

Citation for the published paper:

Tarasov, M. ; Kuzmin, L. ; Otto, E. (2009) "Fabricationtion and Characteristics of Mesh Band-Pass Filters". Instruments and Experimental Techniques, vol. 52(1), pp. 74-78.

http://dx.doi.org/10.1134/S0020441209010114

Downloaded from: http://publications.lib.chalmers.se/publication/88446

Notice: Changes introduced as a result of publishing processes such as copy-editing and formatting may not be reflected in this document. For a definitive version of this work, please refer to the published source. Please note that access to the published version might require a subscription. 


\title{
ELECTRONICS \\ AND RADIO ENGINEERING
}

\section{Production and Characteristics of Grid Band-Pass Filters}

\author{
M. A. Tarasov ${ }^{a}$, V. D. Gromov ${ }^{b}$, G. D. Bogomolov ${ }^{c}$, E. A. Otto ${ }^{d}$, and L. S. Kuz'min ${ }^{e, f}$ \\ ${ }^{a}$ V.A. Kotel'nikov Institute of Radio Engineering and Electronics, Russian Academy of Sciences, \\ ul. Mokhovaya 11, korp. 7, Moscow, 125009 Russia \\ e-mail: tarasov@hitech.cplire.ru \\ ${ }^{b}$ Institute of Space Research, Russian Academy of Sciences, ul. Profsoyuznaya 84/32, Moscow, 117997 Russia \\ ${ }^{c}$ P.L. Kapitsa Institute for Physical Problems, Russian Academy of Sciences, ul. Kosygina 2, Moscow, 119334 Russia \\ ${ }^{d}$ Oxford University, Denys Wilkinson Building, Keble Road, Oxford, OX1 3RH England, United Kingdom \\ ${ }^{e}$ Chalmers University of Technology, Gothenburg, SE-41296 Sweden \\ ${ }^{f}$ D.V. Skobel'tsyn Research Institute of Nuclear Physics, Moscow State University, Moscow, 119992 Russia \\ Received May 7, 2008; in final form, July 9, 2008
}

\begin{abstract}
Grid band-pass filters for 300-, 450-, 600-, and 750-GHz central frequencies are designed and manufactured. Copper and aluminum foil and foil-clad fluoroplastic filters were made by chemical and ionic etching methods, and an aluminum film sputtered on a kapton film was formed by the lift-off lithography method. A gold layer was electrolytically applied on copper grid filters. Characteristics of the filters were measured in the millimeter, submillimeter, and infrared (IR) ranges. Foil filters demonstrate better characteristics at lower frequencies, while filters with sputtered films have better characteristics at upper frequencies. The smallest transmission loss was $0.13 \mathrm{~dB}$. For a stage consisting of four filters, this loss was $0.9 \mathrm{~dB}$. The IR radiation attenuation in a 2.5 - to $25-\mu \mathrm{m}$ wavelength region was no less than $11 \mathrm{~dB}$ per filter.
\end{abstract}

PACS numbers: 07.57.-n

DOI: $10.1134 / \mathrm{S} 0020441209010114$

\section{INTRODUCTION}

At present, microbolometers cooled down to ultralow temperatures $(T<1 \mathrm{~K})$ are finding increasing use in astrophysical studies (radiometry at frequencies over $100 \mathrm{GHz}$ ). To prevent the overheating of cryogenic receiving devices from external thermal radiation, it is necessary to use cooled band-pass filters. In cryostats with pumped-out ${ }^{3} \mathrm{He}$ vapors and a temperature of $\sim 300 \mathrm{mK}$, the background infrared (IR) room-temperature radiation should be attenuated by more than three orders of magnitude, and in dilution cryostats with a base temperature of $\sim 30 \mathrm{mK}$, by more than four orders of magnitude. It is impossible to achieve this attenuation with one filter. In practice, band-pass filters are installed on available radiation screens of a cryostat at $T=100$ and $4 \mathrm{~K}$ and 300 and $100 \mathrm{mK}$. In this case, it is desirable to minimize the introduced attenuation at the signal frequency. Simultaneously, these filters should form operating spectral bands specified by observation aims, since, in contrast to receiver-mixers, bolometers do not naturally have intermediate-frequency filters.

As band-pass filters, resonance metal-grid filters [1] or interference filters based on them [2] are often used at frequencies from hundreds of gigahertz to units of terahertz. Grids are flat periodic structures made of metal on a dielectric substrate or without it. They are sometimes called frequency-selective surfaces (FSSs).

The application area of these filters has been lately expanded to the IR and visible spectrum regions in a hundreds-of-terahertz frequency band [3]. This expansion is largely related to the replacement of the technology. The grid filters initially manufactured on the equipment of the opticomechanical industry and vacuum electronics $[4,5]$ were later replaced by grids of more complex shapes [6-9] created by micro- and nanostructure technologies, which became more accessible.

\section{DESIGN AND MANUFACTURE OF FILTERS}

While designing the filters, we used more easy-tomanufacture thin grids, the sheet thickness of which is much smaller than resonance wavelength $\lambda_{0}$. These filters are scaled well; i.e., when the period is changed, the spectral characteristic remains unchanged under variations of the scale of wavelengths in proportion to the period. Inductive grids with holes in the form of crosses were selected for the band-pass filters (Fig. 1).

According to the terminology of Ulrich [1], the grids, the openings of which are connected by conductive links, are inductive. These grids can be both on a dielectric and without it (free-standing grids) in contrast to grids with insulated openings (capacitive), which always need substrates. The selection of cross parameters $L$ and $W$ (see Fig. 1) allows one to obtain a filter with required quality factor $Q=\lambda_{0} /\left(\lambda_{1}-\lambda_{2}\right)$, where $\lambda_{1}$ and $\lambda_{2}$ are two wavelengths corresponding to a $50 \%$ transmission from the maximum $(-3 \mathrm{~dB})$. The reasonable selection of the parameters also allows one to 
obtain a passband without an absorption line (the socalled Wood anomaly) at the wavelength equal to the period $(\lambda=P)$. In this respect, cross openings are more preferable than square or circular, which are typical of the former technologies.

In the first approximation, the band-pass characteristic of the grid filter is described by the Ulrich theory [1], according to which a grid hole is considered a diaphragm in a waveguide and described as a parallel oscillatory $L C$ circuit brought into a transmission line with characteristic impedance $Z_{0}$ equal to the wave impedance of the free space. This approximation has sense in a single-mode region of the waveguide and, hence, is not applicable to waves that are both too long and too short $(\lambda>>P$ and $\lambda<<P)$.

The ohmic loss is described by resistance $R$ connected to the circuit in series with $L$. For foil grids, the order of $R$ magnitude is close to the surface metal resistance determined by the thickness of the skin layer at frequency $f_{0}=c / \lambda_{0}$. Since, for good conductors, $R<<Z_{0}$, quality factor $Q$ is determined by the ratio $Z_{0} / 2 \pi f_{0} L$ and does not almost depend on $R$. Hence, when scaling the grids, it is possible to neglect the ohmic losses.

The role of the ohmic losses for metal-film grids on substrates is much more significant owing to the fact that the metal thickness of these grids is not as large as that of foils. Grids on polymer substrates cannot be annealed, and, hence, measured values $R$ are much larger than those determined from formulas for a solid metal, even when the metal film is several times thicker than the skin layer. Owing to the ohmic losses, the maximum of the pass-band curve differs from unity. Losses of these filters decrease on cooling, but, in this case, the quality factor of the filter slightly increases.

To calculate the spectra of the grid filters, commercial packages of electromagnetic simulation programs are widely used (such as Microstrip from Sonnet Co., HFSS from Ansoft Co., and others). In spite of the fact that these programs are complex, expensive, and require significant computational resources, the comparison of the calculation and experiment shows (see, e.g., [10]) that the accuracy of these calculations is lower than that expected while scaling the grids.

There are analytic expressions for the resonance wavelength of the grid filters with crossed holes (see, e.g., [10]). According to the theoretical calculation as a part of the simple dipole interaction model,

$$
\lambda_{0}=2 L-W,
$$

while the approximation [10] of the electromagnetic simulation results under the A. Golden (Chicago University) program gives

$$
\lambda_{0}=2 L-1.35 \mathrm{~W}+0.2 B,
$$

where $B=P-L$ is the width of the jump between ends of crossed holes.

The analysis of the experimentally measured data $[6,10-14]$ on grids with various cross parameters has

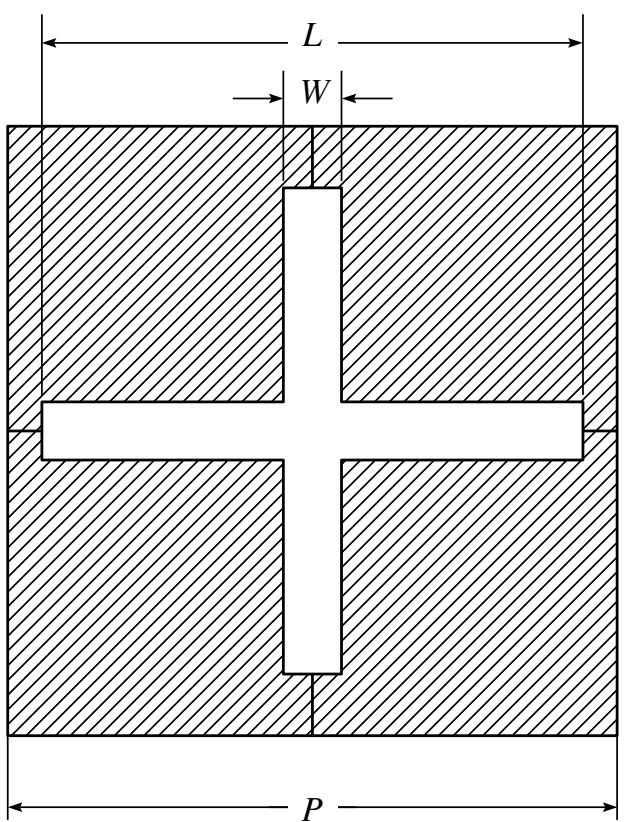

Fig. 1. Drawing of the unit cell of the band-pass filter.

shown that there is a significant deviation of the dependence $\lambda_{0}(L, B, W)$ from the exceeding the difference between (1) and (2). Based on the analysis results, it is possible to conclude that, when $L>0.6 P$, it is possible to use the following half-wave dipole approximation with an accuracy of $\sim 5 \%$ for grids without substrates:

$$
\lambda_{0}=c / f_{0}=2 L \text {. }
$$

A higher accuracy can be obtained by scaling measured grids, data on which can be taken from references or obtained on pilot samples.

Quality factor $Q$ depends on relative sizes of the cross $(P: L: W)$. In particular, when $P: L: W=10$ : $8: 1, Q=2.6$, and, for other parameters, it may reach $\sim 10$ [11].

We used expression (3) for $f_{0}=300,450,600$, and $750 \mathrm{GHz}$, and masks of pilot samples were made with a ratio of the period, length, and width $P: L: W=10$ : $8: 1$.

The maxima of spectral characteristics may differ from $f_{0}$ due to not only an inaccuracy of expression (3). In the absence of a substrate, one has to make grids thicker. In this case, the grid thickness becomes important. In particular, the grid geometry differs from the mask. The grids on a substrate usually have a submicron thickness, and their geometry corresponds to the mask with a high accuracy. However, it is necessary to take into account the influence of the substrate, in particular the difference of the wavelength in a dielectric from that in vacuum. If the grid is completely submerged into the dielectric, as it is sometimes done for interference filters, then $\lambda_{\text {eff }}=\lambda_{0} / \sqrt{\varepsilon}$, where $\varepsilon$ is the per- 


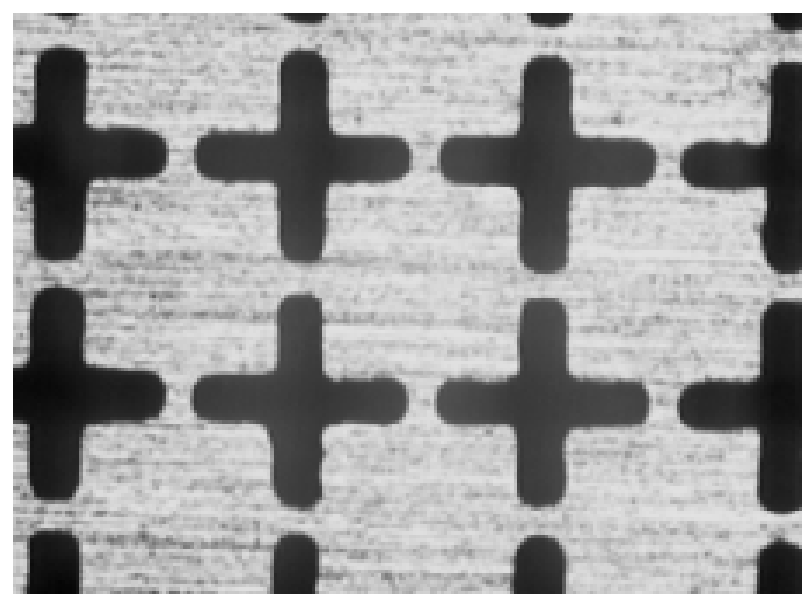

Fig. 2. Microphotograph of the $300-\mathrm{GHz}$ filter made of a foiled fluoroplastic by chemical etching with the further electrolytic gold covering. The cross length $L=500 \mu \mathrm{m}$.

mittivity. For a one-sided substrate, $\lambda_{\text {eff }}=\lambda_{0} / \sqrt{\varepsilon_{\text {eff }}}, 1<$ $\varepsilon_{\text {eff }}<(\varepsilon+1) / 2$. In this case, $\varepsilon_{\text {eff }} \approx(\varepsilon+1) / 2$ is already at dielectric thickness $t=0.1 \lambda_{0}$ [6]. The reflection from the substrate surface and its interference with the reflection from the grid on the second surface also affects the spectral characteristic.

Filters of several types were manufactured by methods of chemical etching of a copper foil $50 \mu \mathrm{m}$ thick, an aluminum foil $20 \mu \mathrm{m}$ thick, foil-clad fluoroplastic with a 35- $\mu \mathrm{m}$ copper foil thickness and a $0.8-\mathrm{mm}$ fluoroplastic substrate thickness, by chemical and ionic etching of an aluminized mylar, and thermal sputtering of an aluminum film $0.4 \mu \mathrm{m}$ thick. An electrolytic gold layer coating was used to eliminate the copper foil oxidation.

The pattern was formed by the contact optical lithography method, illuminating an S1813 photoresist through one of chromium photomasks made by the electronic lithography. Upon the development, the copper was etched in a hydrogen peroxide- and hydrochloric acid-based solution and the aluminum was etched in an orthophosphoric acid-based solution or their ion etching was performed in the CAIBE Oxford Ionfab setup. Figure 2 shows a photograph of one manufactured filter.

\section{MEASUREMENT RESULTS}

Characteristics of the filters were measured by the Epsilon spectrometer (IOFAN design) based on a backward-wave tube (BWT) in a 120 - to $380-\mathrm{GHz}$ region, by a Beckman IR-720M Fourier-transform spectrometer in a $200-\mathrm{GHz}$ to $1.5-\mathrm{THz}$ region, and by a Shimadzu IR-460 spectrometer in a 2.5- to $25-\mu \mathrm{m}$ IR wave range. Examples of spectral characteristics of the filters are given in Figs. 3 and 4.

Foil filters have demonstrated better characteristics at lower frequencies $f_{0}$, and filters sputtered on a Mylar $20 \mu \mathrm{m}$ thick or kapton $40 \mu \mathrm{m}$ thick have shown better characteristics at higher frequencies. The lowest loss

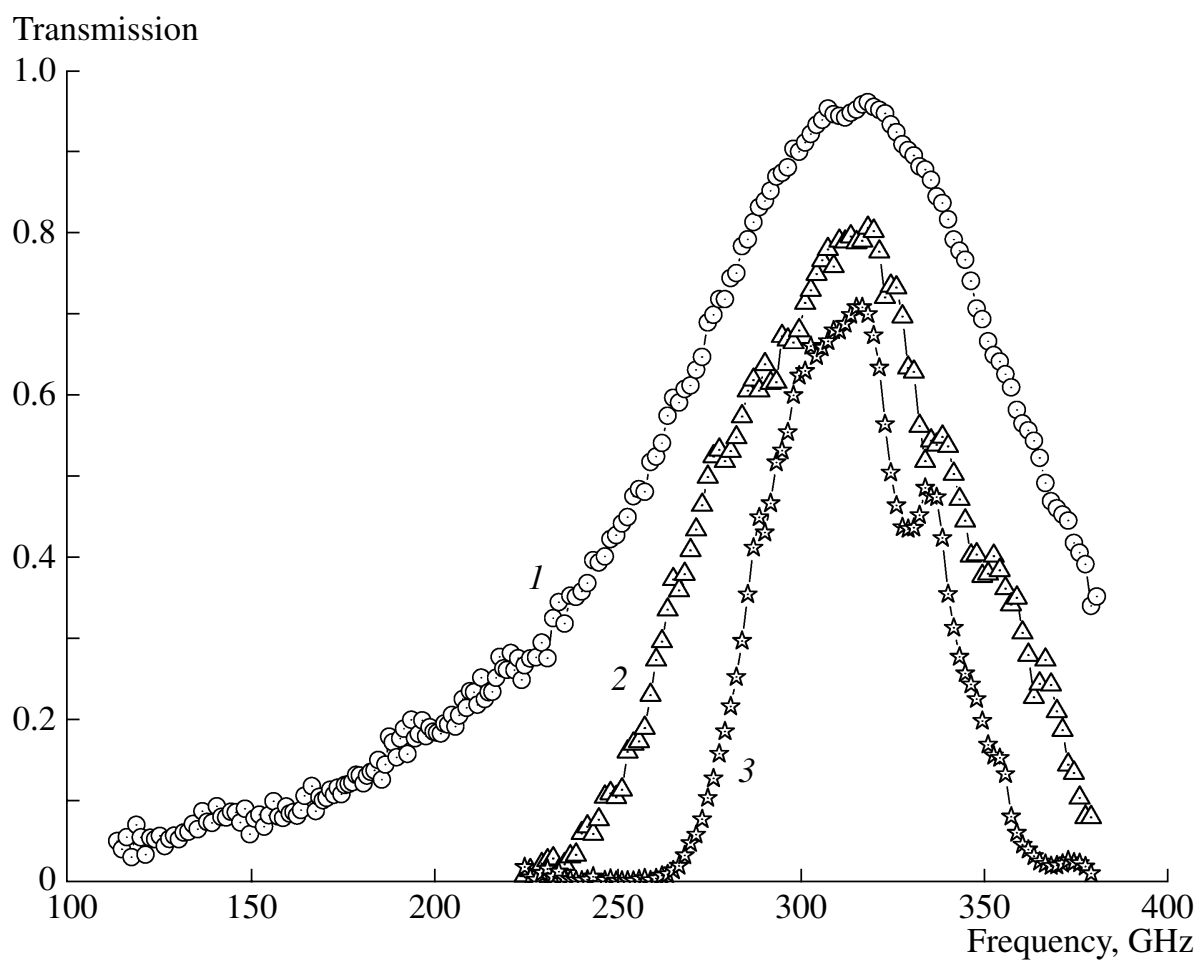

Fig. 3. Transmission spectra of the $300-\mathrm{GHz}$ filters made of an aluminum foil: $(I)$ one filter with a peak transmission of 0.96 , (2) three filters with a peak transmission of 0.81 , and $(I)$ four filters with a peak transmission of 0.71 . 
Transmission

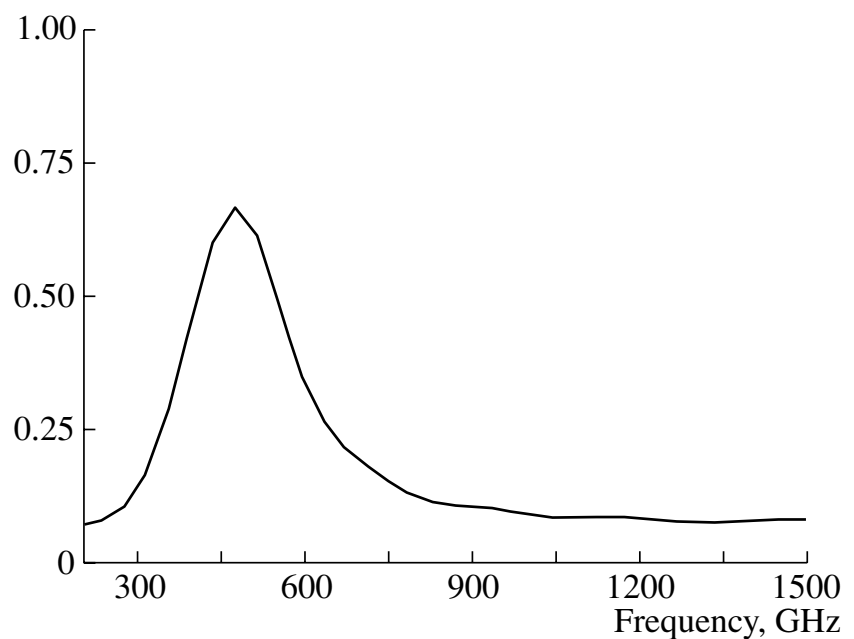

Fig. 4. Transmission spectrum of the copper filter on a Mylar film.

values at the transmission maximum were $0.13 \mathrm{~dB}$ for the foil filters. For a stage (set) of four filters, the losses were $0.9 \mathrm{~dB}$. For a stage of four filters separated by filtered paper sheets, the losses at the center were $1.5 \mathrm{~dB}$; in this case, a specific behavior related to the FabryPerot resonance was observed at band ends. When the resonator is exactly adjusted and tuned, it is possible to significantly improve the quality factor of the filter stage.
Filters on a thick fluoroplastic layer have additional losses at a 1-dB level due to reflections from two airsubstrate interfaces. Filters based on an aluminum film $0.4 \mu \mathrm{m}$ thick, sputtered on kapton, had losses up to $5 \mathrm{~dB}$ at the central frequency. This can be attributed to resistive losses both in a thin aluminum film and a chromium sublayer $5 \mathrm{~nm}$ thick applied for improving adhesion. The losses measured in a photomask, which is a chromium film on a glass substrate, were $20 \mathrm{~dB}$ at the central frequency, visually illustrating the role of resistive losses in the filter material.

As was noted above, operation of cryogenic bolometers is only possible at a strong suppression of the background IR radiation. For this purpose, special cold disabling filters (for example, fluorogold) are used. The attenuation of the IR background by a band-pass filter outside its working band reduces requirements for disabling filters. The band-pass spectra of the grid filters in the 2.5- to $25-\mu \mathrm{m}$ IR wavelength range are shown in Fig. 5. Deep transmission minima at 6 and $13 \mu \mathrm{m}$ relate to reflections and absorptions in the Mylar film, on which this filter is based. The filters can be efficiently staged in the IR wave range in contrast to the submillimeter range, since the mutual darkening of holes of subsequent grids leads to the fact the total transmission is much smaller than products of transmissions of single filters. In our spectrometer, the signal transmitted through two filters was below the noise level, i.e., the attenuation exceeded $20 \mathrm{~dB}$.

The radiation power density at $T=300 \mathrm{~K}$ may reach $46 \mathrm{~mW} / \mathrm{cm}^{2}$; when the window area is $5 \mathrm{~cm}^{2}$, the com-

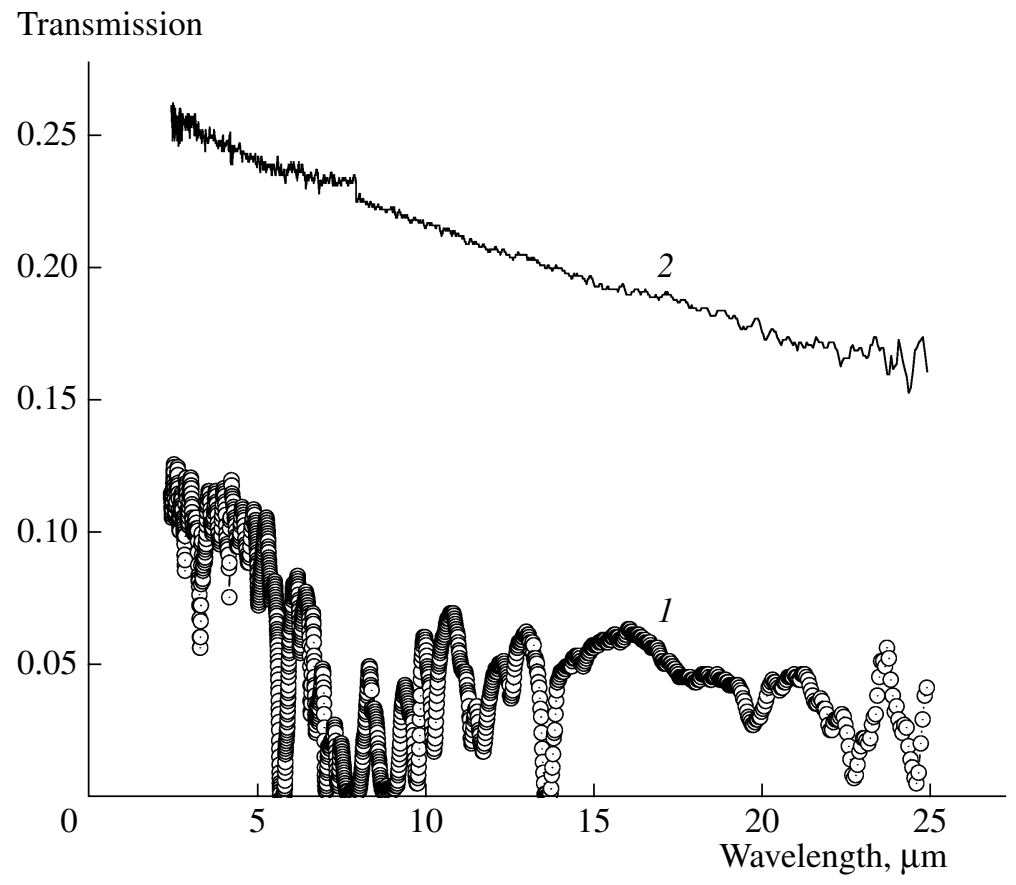

Fig. 5. Transmission spectra in a 2.5- to 25- $\mu \mathrm{m}$ range for filters: (1) on Mylar; (2) 300-GHz filter on an aluminum foil with widened (due to etching) holes. 
plete power through the window may be $220 \mathrm{~mW}$. A HELIOX standard cryostat from the Oxford Instruments Co. with ${ }^{3} \mathrm{He}$ vapor pumping has a cooling power of $\sim 100 \mu \mathrm{W}$ at $300 \mathrm{mK}$ so that the attenuation of the background radiation should be no less than $33 \mathrm{~dB}$, reached by placing three grid filters. A TRITON dilution cryostat from the same company with a cooling power of $20 \mu \mathrm{W}$ per $100 \mathrm{mK}$ requires that four filters be already installed. In this case, the thermal radiation power of the room temperature in a $60-\mathrm{GHz}$ bandwidth must be $k T \Delta f=300 \mathrm{pW}$, setting a supersensitive bolometer to a saturation region. However, for a space background radiation temperature of $2.7 \mathrm{~K}$, the power of the satellite- or balloon-carried receiver in the bandwidth will be already smaller than $3 \mathrm{pW}$, falling within a dynamic range of the superconducting cold-electron bolometer [15].

\section{ACKNOWLEDGMENTS}

We are grateful to V.V. Zav'yalov, I.A. Maslov, E.A. Tishchenko, and L.E. Svistunov for assistance in the work and fruitful discussions.

This study was supported by the Russian Foundation for Basic Research (project no. 05-02-19650), NTsNIL (project no. 08-02-00518), and FANI (project no. 02.513.11.3157).

\section{REFERENCES}

1. Ulrich, R., Infrared Physics, 1967, vol. 7, no. 1, p. 37.

2. Ulrich, R., Appl. Opt., 1968, vol. 7, no. 10, p. 1987.

3. Luker, A., Hein, H., Schulz, J., et al., Infrared Physics Technology, 2008, vol. 51, p. 178.

4. Bravermann, N.R., Vorob'ev, L.V., Soglasnova, V.A., and Sholomitskii, G.B., Preprint of Inst. for Space Research, USSR Acad. Sci., Moscow, 1975, Pr-223.

5. Soglasnova, V.A., Sholomitskii, G.B., Gromov, V.D., et al., Optiko-Mekhanich. Promyshl., 1978, no. 1, p. 75.

6. Mac Donald, M., Alexanin, A., York, R., et al., IEEE Trans. MTT, 2000, vol. 48, no. 4, p. 712.

7. Winnewisser, C., Lewin, F., Schall, M. et al., IEEE Trans. MTT, 2000, vol. 48, no. 4, p. 744.

8. Campos, A., d'Assuncao, A., and de Melo, M., Int. J. IR MM Waves, 2000, vol. 21, no. 3, p. 461.

9. Lima, I. and Giarola, A., Int. J. IR MM Waves, 2000, vol. 21, no. 3, p. 447.

10. Moller, K.D. and Farmer, K.R., Infrared Physics Technology, 1999, vol. 40, p. 475.

11. Chase, S.T. and Joseph, R.D., Appl. Opt., 1983, vol. 22, p. 1775.

12. Porterfield, D.W., Hesler, J.L., Densing, R., et al., Appl. Opt., 1994, vol. 33, p. 6046.

13. Soglasnova, V.A. and Gorshunov, B.P., Int. J. IR $M M$ Waves, 1993, vol. 14, p. 155.

14. Soglasnova, V.A. and Kreysa, E., Gemund, H.-P., and Maslov, I.A., ESA-SP, 1996, vol. 388, p. 95.

15. Tarasov, M., Kuz'min, L., Stepantsov, E., et al., Pis'ma Zh. Eksp. Teor. Fiz., 2004, vol. 79, no. 6, p. 356.

SPELL: OK 